\title{
Review: antibiotics improve maternal and fetal outcomes and are safe in preterm, prelabour rupture of membranes
}

\author{
Kenyon S, Boulvain M, Neilson J. Antibiotics for preterm premature rupture of membranes. Cochrane Database Syst Rev \\ 2002;(1):CD001058 (latest version 23 Aug 2001).

\section{QUESTION: In women with preterm, prelabour rupture of membranes, what are the effectiveness and safety of antibiotics for maternal and fetal outcomes?}

\author{
Source of funding: no \\ external funding. \\ For correspondence: \\ Ms S Kenyon, Leicester \\ Royal Infirmary, \\ Leicester, UK. \\ oracle@le.ac.uk.
}

\section{COMMENTARY}

Preterm birth, with or without rupture of membranes, is a major problem in obstetric and neonatal care. It contributes significantly to maternal morbidity and perinatal morbidity and mortality. Infection has generally been implicated in the cause of preterm labour, but it is of special concern in threatened preterm labour because of ruptured membranes.

Antibiotics for threatened preterm birth are not particularly effective among women with intact membranes. ${ }^{1}$ Clinicians have been ambivalent about the effectiveness of antibiotics among women with ruptured membranes who do not have other signs of infection. The review by Kenyon et al collated and aggregated high quality evidence, and from its meta-analyses we can be confident that antibiotics are associated with reduced maternal and neonatal morbidity in preterm, prelabour rupture of membranes. However, this message comes with a warning about $\beta$-lactam antibiotics.

Can we believe that $\beta$-lactam antibiotics increase the risk for necrotising enterocolitis? Subgroup analyses should be interpreted with caution. By reducing the number of trials per subgroup, such analyses run into problems with reduced power, risking an inability to detect a difference when one exists (type II error). However, by increasing the number of comparisons in a review, problems with spurious significance may arise, thereby risking detection of differences that do not exist (type I error). We can be certain about the effect observed in a subgroup analysis if a biological rationale for the effect exists, the effect is large, the subgroup analysis is planned in advance, and the analysis is one of a small number of subgroup analyses. Answers to these questions may not be immediately apparent. In summary, the safest inference is that macrolide antibiotics should be recommended for patients with threatened preterm labour with ruptured membranes.

Khalid S Khan, MBBS, MSc Birmingham Women's Hospital Birmingham, UK

1 King J, Flenady V. Antibiotics for preterm labour with intact membranes. Cochrane Database Syst Rev 2002;(2):CD000246.

\section{Study selection}

Studies were selected if they were randomised controlled trials, compared antibiotic use with placebo or different antibiotic regimens, included women with preterm (defined as $<37 \mathrm{wk}$ ) rupture of membranes, and reported clinically relevant outcomes.

\section{Data extraction}

Data were extracted on antibiotic type, outcomes, and study quality.

\section{Main results}

32 trials were identified, and 13 trials that randomised $>6000$ women and their babies were included in the review. Most trials were small except for 2 large trials (1 with 4826 and 1 with 614 women). Women were recruited between 20 and 37 weeks of gestation, and most women were not in active labour. 9 trials tested broad spectrum penicillin alone or in combination, 5 tested $\beta$-lactam antibiotics alone or in combination, and 1 tested clindamycin and gentamycin. 5 trials used oral antibiotics alone, 2 used intravenous antibiotics alone, and 6 used a combination of oral and intravenous antibiotics. Any antibiotic, especially a macrolide antibiotic, was associated with greater improvements in maternal and fetal outcomes than placebo (table). $\beta$-lactam antibiotics were associated with greater neonatal necrotising enterocolitis risk than was placebo (table). No evidence existed for major adverse drug reactions.

\section{Conclusions}

In women with preterm, prelabour rupture of membranes, antibiotics are generally safe and improve maternal and fetal outcomes. Macrolide antibiotics are associated with improved outcomes. $\beta$-lactam antibiotics are associated with increased neonatal necrotising enterocolitis.

Any antibiotic v placebo in preterm, prelabour rupture of membranes*

\begin{tabular}{|c|c|c|c|}
\hline & Weighted event rates & & \\
\hline Outcomes & Any antibiotic $v$ placebo & $\operatorname{RRR}(95 \% \mathrm{Cl})$ & NNT (Cl) \\
\hline Maternal infection after delivery and before discharge & $18 \%$ v $20 \%$ & $15 \%(4$ to 24$)$ & 33 (19 to 143$)$ \\
\hline Chorioamnionitis & $16 \%$ v $26 \%$ & $38 \%(25$ to 49$)$ & 11 (8 to 17$)$ \\
\hline Birth within 24 hours & $32 \% \vee 40 \%$ & $23 \%(17$ to 28$)$ & $12(9$ to 16$)$ \\
\hline Birth within 7 days & $8 \% \vee 67 \%$ & $12 \%(8$ to 16$)$ & $13(10$ to 19$)$ \\
\hline Neonatal infection, including pneumonia & $11 \% \vee 17 \%$ & $33 \%(15$ to 48$)$ & $18(12$ to 46$)$ \\
\hline Positive neonatal blood culture & $6.4 \% \vee 8.4 \%$ & $25 \%(7$ to 40$)$ & $48(27$ to 200$)$ \\
\hline Neonatal oxygenation $>28$ days & $8.5 \% v 11 \%$ & $19 \%$ (3 to 32$)$ & $48(27$ to 334$)$ \\
\hline \multirow[t]{2}{*}{ Abnormal cerebral ultrasonographic scan before discharge } & $5.6 \% \vee 9.4 \%$ & $18 \%(1$ to 32$)$ & 67 (35 to 1000$)$ \\
\hline & Macrolide antibiotics $v$ placebo & & \\
\hline \multirow[t]{2}{*}{ Birth within 48 hours } & $35 \%$ v $41 \%$ & $16 \%(7$ to 24$)$ & 16 (10 to 35$)$ \\
\hline & $\beta$-Lactam antibiotics $v$ placebo & RRI (Cl) & NNH (Cl) \\
\hline Neonatal necrotising enterocolitis & $2.4 \% \vee 0.48 \%$ & $360 \%$ (98 to 972$)$ & 53 (36 to 112$)$ \\
\hline
\end{tabular}

*Abbreviations defined in glossary; RRR, RRI, NNT, NNH, and Cl calculated from data in article using fixed effects. 